\title{
Super-Helices for Predicting the Dynamics of Natural Hair
}
Florence Bertails
Basile Audoly
Marie-Paule Cani
Bernard Querleux
Frédéric Leroy
Jean-Luc Lévêque
EVASION*/INRIA
$\mathrm{LMM}^{\dagger} / \mathrm{CNRS}$
EVASION*/INRIA
L'Oréal Recherche
L'Oréal Recherche
France
France
L'Oréal Recherche
France
Paris, France
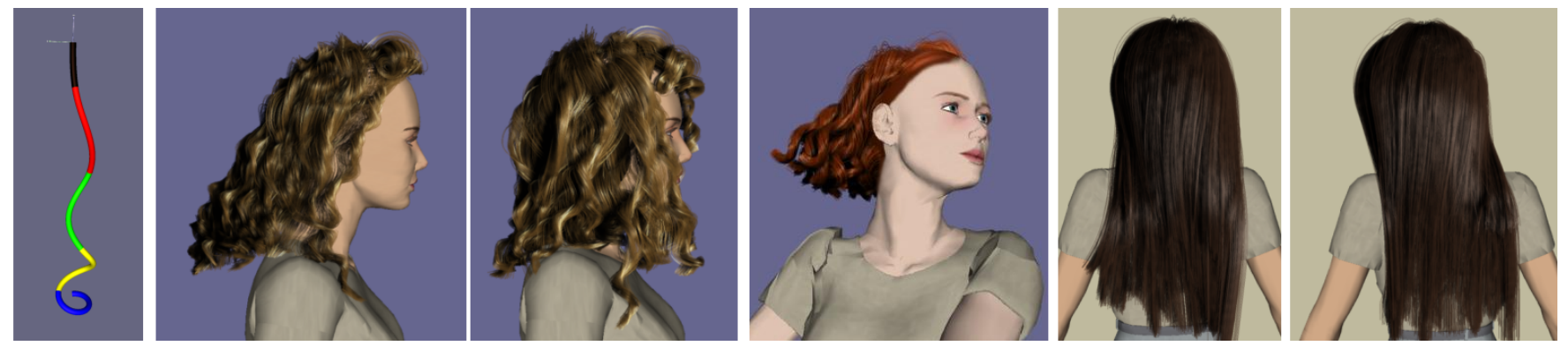

Figure 1: Left, a Super-Helix. Middle and right, dynamic simulation of natural hair of various types: wavy, curly, straight. These hairstyles were animated using $N=5$ helical elements per guide strand.

\begin{abstract}
Simulating human hair is recognized as one of the most difficult tasks in computer animation. In this paper, we show that the Kirchhoff equations for dynamic, inextensible elastic rods can be used for accurately predicting hair motion. These equations fully account for the nonlinear behavior of hair strands with respect to bending and twisting. We introduce a novel deformable model for solving them: each strand is represented by a Super-Helix, i.e., a piecewise helical rod which is animated using the principles of Lagrangian mechanics. This results in a realistic and stable simulation, allowing large time steps. Our second contribution is an in-depth validation of the Super-Helix model, carried out through a series of experiments based on the comparison of real and simulated hair motions. We show that our model efficiently handles a wide range of hair types with a high level of realism.
\end{abstract}

CR Categories: I.3.7 [Computer Graphics]: Three-Dimensional Graphics and Realism-Animation

Keywords: Hair modeling, Physically-based simulation, Cosserat model, Lagrangian dynamics

\section{Introduction}

Realistic hair simulation is one of the most difficult issues when animating virtual humans. Human hair is a very complex material, consisting of hundreds of thousands of very thin, inextensible strands that interact with each other and with the body. Unlike solids or fluids, which have been studied for over a century and well modeled by now classical equations, hair remains a largely

${ }^{*}$ EVASION is a joint research project of CNRS, INPG, INRIA and UJF.

${ }^{\dagger}$ LMM is a joint research lab of Univ. Pierre et Marie Curie and CNRS. unsolved problem described by no well accepted model. Finding a representation that provides an accurate simulation of hair motion remains a challenge.

Modeling hair dynamics raises a number of difficulties. First, each individual strand has a complex nonlinear mechanical behavior, strongly related to its natural shape: smooth, wavy, curly or fuzzy. Second, the dynamics of an assembly of strands takes on a collective behavior; however, to our knowledge there is no quantified data regarding hair clustering and cohesion. Last, the simulation of a full head of hair raises obvious issues in terms of efficiency.

This paper tackles the problem of accurately modeling hair dynamics while keeping computational costs reasonable. Unlike prior work in hair animation, we set up our model using studies from the field of cosmetics on the physical properties of real hair. This allows us to parameterize virtual hair from measured features, yielding realistic simulations that we validated through a set of experiments.

\subsection{Previous work}

Three families of computational models have been used for simulating the dynamics of individual hair strands: Mass-spring systems [Rosenblum et al. 1991; Plante et al. 2001], two dimensional projective dynamics [Anjyo et al. 1992; Daldegan et al. 1993; Lee and Ko 2001], and chains of rigid bodies [Hadap and MagnenatThalmann 2001; Chang et al. 2002]. The first family of models is well suited for animating long, extensible wisps of hair. The second efficiently handles non-stretchable straight hair. The last increases realism by handling the twisting motion of hair strands while still preventing stretching. Although these models have led to very nice visual results, several important hair features have not yet been taken into account. For instance, the deformations of curly hair are quite specific compared to straight hair, but previous work has simply simulated straight extensible hair wisps, with curly strands used only for rendering. Moreover, none of the previous models demonstrated nonlinear behaviors such as bending-twisting instabilities or buckling, which have a great impact on realism.

An accurate mechanical representation for hair strands is the Kirchhoff model [Audoly and Pomeau 2006], which relies on the notion of Cosserat curves. This model was first introduced to the CG community by Pai [2002] who used an iterative integration in space to 
get the statics of a surgical wire. In our previous work, we applied this model to hair, using energy minimization to compute the equilibrium position of strands submitted to gravity and collision forces [Bertails et al. 2005b]. The geometric features of strands such as curliness were accurately modeled, providing a predictive method for a variety of natural hairstyles. However, this approach does not handle dynamics, making it of no use for hair animation. To our knowledge, simulating the dynamics of Cosserat rods has never been addressed in computer graphics. A major contribution of this paper is a robust solution to this difficult problem.

For efficient hair animation, some assumptions need to be made regarding the collective behavior of hair. Hair has been either approached as a continuous medium [Daldegan et al. 1993; Hadap and Magnenat-Thalmann 2001; Chang et al. 2002; Bando et al. 2003] or as a set of disjoint hair wisps [Plante et al. 2001; Koh and Huang 2001; Bertails et al. 2003; Ward and Lin 2003; Choe et al. 2005]. Both approaches render hair from the simulation of a limited number of guide strands. The former uses interpolation to add extra strands at the rendering stage, while the latter extrapolates the positions of the wisp skeletons. Methods based on a continuum are appropriate for smooth and fluid hair, while wispbased approaches achieve their best results for wavy or curly hair. In the two approaches, self interactions are treated in dramatically different ways. For instance, Hadap's continuum model relies on a fluid-like internal viscosity to model hair friction and prevent self-intersections, whereas Plante sets up an anisotropic collision response between hair wisps. None of these models accounts for the specific state of the surface of hair strands when computing collision response, and no attention is paid to modeling the variations of collective hair behavior due to straight, curly or fuzzy types.

We specifically avoid making a choice between continuous and wisp-based approaches by introducing a unified formulation. This allows the collective behavior of hair to be parameterized from observed geometric features whose effects on hair motion are modeled through an adequate tuning of hair interactions.

\subsection{Overview}

This paper presents a novel mechanical model for hair, dedicated to the accurate simulation of hair dynamics. In the spirit of work by Marschner et al. [2003] in the field of hair rendering, we rely on the structural and mechanical features of real hair to achieve realism. This leads us to use Kirchhoff equations for dynamic rods. These equations are integrated in time thanks to a new deformable model that we call Super-Helices: A hair strand is modeled as a $C^{1}$ continuous, piecewise helical ${ }^{1}$ rod, with an oval to circular cross section. We use the degrees of freedom of this inextensible rod model as generalized coordinates, and derive the equations of motion by Lagrangian mechanics. As our validations show, the resulting model accurately captures the nonlinear behavior of hair in motion, while ensuring both efficiency and robustness of the simulation.

Our second contribution is an in-depth validation of the model with respect to hair dynamics, contacts, and collective hair behavior. As our results show, our method can reproduce typical nonlinear effects of hair dynamics, as well as cohesion between neighboring hair clumps. Finally, our model is able to handle a wide range of natural hair types: straight, wavy and curly. Meanwhile, computations are no more expensive than those of the recent hair models presented in computer graphics.

${ }^{1} \mathrm{~A}$ helix is a curve with constant curvatures and twist. Note that this definition includes straight lines (zero curvatures and twist), so Super-Helices can be used for representing any kind of hair.

\section{Hair structure and mechanics}

Achieving realistic simulations of hair motion requires some understanding of hair structure. This section gives a summary of the existing knowledge on hair, mostly issued from the field of cosmetics. Further details can be found in [Lindelof et al. 1988; Robbins 2002].

\subsection{Individual strands}

A human hair fiber is a thin structure (about $0.1 \mathrm{~mm}$ in diameter) with either a circular or oval cross section. The active part, called the follicle, is located under the skin and produces the keratin proteins that compose the hair material. The second part, the visible and dead - part of hair, is called the hair shaft, and corresponds to the "hair strand" we are seeking to animate.

The hair shaft is entirely synthesized by the associated follicle, which acts as a mold for shaping the strand [Lindelof et al. 1988]. It thus has almost uniform cross section, natural twist and natural curvatures all along. These geometric parameters are associated with commonsense notions of straight, curly, or fuzzy hair. Their values are characteristic of the ethnic group from which the hair comes [Robbins 2002]. Africans have follicles with a helical form and an oval cross section, whereas Asians have follicles that are completely straight with a larger and circular cross section. As a result, Asian hair is thicker, with no natural curliness. It makes it look smooth and regular. In contrast, African hair looks frizzy and irregular. Caucasian hair stands between these two extremes.

The internal structure of the shaft consists of three concentric layers from the core to the periphery: a central canal called medulla; the cortex, i.e. cells filled with keratin, contributing $90 \%$ of the total weight; and the cuticle, a thin coating covered by tilted scales. Keratin is a remarkably stiff material, making the shaft extremely difficult to shear and stretch. However, because its cross section is very small, it can be easily bent and twisted.

Deformations of a hair strand involve rotations that are not infinitely small and so can only be described by nonlinear equations [Audoly and Pomeau 2006]. Physical effects arising from these nonlinearities include instabilities called buckling. For example, when a thin hair wisp is held between two hands that are brought closer to each other (see Figure 2, right), it reacts by bending in a direction perpendicular to the applied compression. If the hands are brought even closer, a second instability occurs and the wisp suddenly starts to coil (the bending deformation is converted into twist).
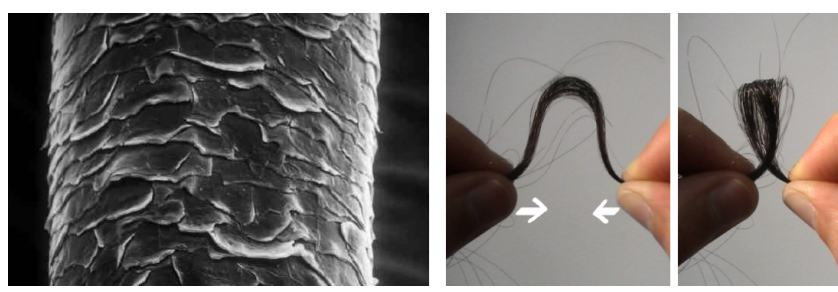

Figure 2: Left, close view of a hair fiber (root upwards) showing the cuticle covered by overlapping scales. Right, bending and twisting instabilities observed when compressing a small wisp.

\subsection{Collective behavior of hair}

Hair is composed of around 150,000 strands that interact with each other and with the body. Hair contacts have a complex nature due 
to the tilted scales that pave the cuticle of the hair shaft (see Figure 2, left). This irregular surface causes anisotropic friction forces, whose amplitude strongly depends on the direction of sliding with respect to the orientation of the scales. This can be easily felt by rubbing a fiber between thumb and forefinger. Due to the lightness of a strand, these friction forces are much higher than other external forces. This explains the strong cohesion observed in hair motion.

The type and shape of strands have a significant effect on collective hair behavior. The most obvious example of this is hair curliness. Hair clumps are more likely to appear in curly hair, where the greater number of contacts among hair strands increases the probability that they will become entangled. In contrast, strands of straight hair slide easily with their neighbors, and so they appear as a continuum rather than as a set of disjoint wisps. In general, as the complexity of hair geometry increases, there is more internal friction and fewer available degrees of freedom for motion. Modeling these features is essential for yielding realistic motion of a full head of hair, but it remains a challenging issue due to the lack of quantitative studies on hair clustering.

\section{Super-Helices}
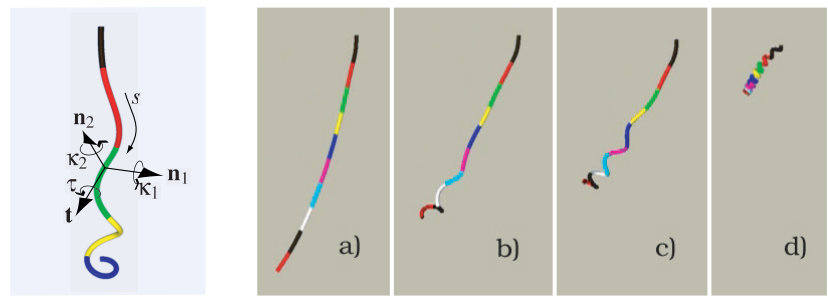

Figure 3: Left, geometry of Super-Helix. Right, animating SuperHelices with different natural curvatures and twist: a) straight, b) wavy, c) curly, d) strongly curly. In this example, each Super-Helix is composed of 10 helical elements.

We shall first present the model that we used to animate individual hair strands (guide strands). This model has a tunable number of degrees of freedom. It is built upon the Cosserat and Kirchhoff theories of rods. In mechanical engineering literature, a rod is defined as an elastic material that is effectively one dimensional: its length is much larger than the size of its cross section.

\subsection{Kinematics}

We consider an inextensible rod of length $L$. Let $s \in[0, L]$ be the curvilinear abscissa along the rod. The centerline, $\mathbf{r}(s, t)$, is the curve passing through the center of mass of every cross section. This curve describes the shape of the rod at a particular time $t$ but it does not tell how much the rod twists around its centerline. In order to keep track of twist, the Cosserat model introduces a material frame $\mathbf{n}_{i}(s, t)$ at every point of the centerline ${ }^{2}$. By material, we mean that the frame 'flows' along with the surrounding material upon deformation. By convention, $\mathbf{n}_{0}$ is the tangent to the centerline:

$$
\mathbf{r}^{\prime}(s, t)=\mathbf{n}_{0}(s, t),
$$

while $\left(\mathbf{n}_{\alpha}\right)_{\alpha=1,2}$ span the plane of the cross section, see Figure 3, left. We use primes to denote space derivatives along the center line, $f^{\prime}=\partial f / \partial s$, while the overstruck notation is for time derivatives, $\dot{f}=\mathrm{d} f / \mathrm{d} t$.

\footnotetext{
${ }^{2}$ By convention, lowercase Latin indices such as $i$ are used for all spatial directions and run over $i=0,1,2$ while Greek indices such as $\alpha$ are for spatial directions restricted to the plane of the cross section, $\alpha=1,2$.
}

The Kirchhoff model for elastic rod starts from this mathematical description of a Cosserat curve and adds the physical requirement of inextensibility and unshearability. In this case, the frame $\left(\mathbf{n}_{i}(s)\right)_{i=0,1,2}$ is orthonormal for all $s$, and there exists a vector $\boldsymbol{\Omega}(s, t)$, called the Darboux vector, such that:

$$
\mathbf{n}_{i}^{\prime}(s, t)=\boldsymbol{\Omega}(s, t) \times \mathbf{n}_{i}(s, t) \quad \text { for } i=0,1,2 .
$$

Appropriate boundary conditions must be specified: one end of the hair strand, $s=0$, is clamped into the head while the other end, $s=L$, is free. The position of the clamped end, together with the orientation of the initial frame, are imposed by head motion (an input in the simulations):

$$
\left\{\begin{array}{l}
\mathbf{r}(0, t)=\mathbf{r}_{\mathrm{c}}(t) \\
\mathbf{n}_{i}(0, t)=\mathbf{n}_{i, \mathrm{c}}(t) \quad \text { for } i=0,1,2,
\end{array}\right.
$$

where subscript 'c' refers to the clamped end of the rod, $s=0$.

The rod's material curvatures $\left(\kappa_{\alpha}(s, t)\right)_{\alpha=1,2}$ with respect to the two directions of the cross section and the twist $\tau(s, t)$ are defined as the coordinates of the vector $\boldsymbol{\Omega}(s, t)$ in the local material frame:

$$
\boldsymbol{\Omega}(s, t)=\tau(s, t) \mathbf{n}_{0}(s, t)+\kappa_{1}(s, t) \mathbf{n}_{1}(s, t)+\kappa_{2}(s, t) \mathbf{n}_{2}(s, t) .
$$

By introducing a redundant notation for the twist, $\kappa_{0}=\tau$, we can refer to these parameters collectively as $\left(\kappa_{i}(s, t)\right)_{i=0,1,2}$.

\subsection{Reconstruction, generalized coordinates}

The degrees of freedom of a Kirchhoff rod are its material curvatures and twist $\left(\kappa_{i}(s, t)\right)_{i=0,1,2}$. A continuous model being of little use for computer animation, we introduce a spatial discretization as follows. Let us divide the strand $s \in[O, L]$ into $N$ segments $S_{Q}$ indexed by $Q(1 \leq Q \leq N)$. These segments may have different lengths, and $N$ is an arbitrary integer, $N \geq 1$. We define the material curvatures and twist of our deformable model with piecewise constant functions over these segments. We write $q_{i, Q}(t)$ the constant value of the curvature $\kappa_{i}$ (for $i=1,2$ ) or twist $\kappa_{0}=\tau$ (for $i=0$ ) over the segment $S_{Q}$ at time $t$. Therefore, an explicit formula for the material curvatures and twist reads

$$
\kappa_{i}(s, t)=\sum_{Q=1}^{N} q_{i, Q}(t) \chi_{Q}(s)
$$

where $\chi_{Q}(s)$ is the characteristic function of segment $Q$, equal to 1 if $s \in S_{Q}$ and 0 otherwise. We collect the numbers $q_{i, Q}(t)$ into a vector $\mathbf{q}(t)$ of size $3 N$, which we call the generalized coordinates of our model.

These generalized coordinates $\mathbf{q}(t)$ can be used to reconstruct the rod shape at any given time. Indeed, plugging equation (3) into equation (2), and then equation (2) into equations (1a-c) yields a differential equation with respect to $s$. By integration of this equation, one obtains the centerline $\mathbf{r}(s)$ and the material frames $\mathbf{n}_{i}(s)$ as a function of $s$ and $\mathbf{q}(t)$. This process, called the reconstruction, can be carried out analytically; as explained in Appendix A, the integration with respect to $s$ has a symbolic solution over every segment $S_{Q}$. By patching these solutions, we find that our model deforms as a helix over every segment $S_{Q}$ and, moreover, is $C^{1}$-smooth (between adjacent helices, both the centerline and the material frames are continuous). This is why we call this model a Super-Helix. We write $\mathbf{r}^{\mathrm{SH}}(s, \mathbf{q})$ and $\mathbf{n}_{i}^{\mathrm{SH}}(s, \mathbf{q})$ as the parameterization of the SuperHelix in terms of its generalized coordinates q. In Appendix A, we explain how these functions $\mathbf{r}^{\mathrm{SH}}$ and $\mathbf{n}_{i}^{\mathrm{SH}}$ can be obtained in symbolic form.

Imposing a uniform value to the material curvatures and twist over the hair length would make it deform as a plain helix. This is indeed what happens when one chooses the coarsest possible spatial 
discretization, that is $N=1$. For other values of $N$, the rod is made of several helices patched together. Large values of $N$ yield arbitrarily fine space discretizations.

\subsection{Dynamic equations for a Super-Helix}

Given a deformable body whose configuration depends on generalized coordinates $\mathbf{q}(t)$, Lagrangian mechanics provides a systematic method for deriving its equations of motion, $\ddot{\mathbf{q}}=\mathbf{a}(\mathbf{q}, \dot{\mathbf{q}}, t)$. This is done by feeding the Lagrangian equations of motion:

$$
\frac{\mathrm{d}}{\mathrm{d} t}\left(\frac{\partial T}{\partial \dot{q}_{i Q}}\right)-\frac{\partial T}{\partial q_{i Q}}+\frac{\partial U}{\partial q_{i Q}}+\frac{\partial D}{\partial \dot{q}_{i Q}}=\int_{0}^{L} \mathbf{J}_{i Q}(s, \mathbf{q}, t) \cdot \mathbf{F}(s, t) \mathrm{d} s
$$

with the expressions for the kinetic energy $T(\mathbf{q}, \dot{\mathbf{q}}, t)$, for the internal energy $U(\mathbf{q}, t)$ and for the dissipation potential $D(\mathbf{q}, \dot{\mathbf{q}}, t)$ that describe the physics of the system at hand. The right-hand side of equation (4) is the generalized force $f_{i Q}$ deriving from the lineic density $\mathbf{F}(s, t)$ of physical force applied on the rod, and $\mathbf{J}_{i Q}$ defines the Jacobian matrix, $\mathbf{J}_{i Q}=\partial \mathbf{r}^{\mathrm{SH}}(s, \mathbf{q}) / \partial q_{i Q}$. We consider three force contributions, namely hair weight, viscous drag from ambient air (considered at rest for simplicity) with coefficient $v$, and interaction forces with surrounding strands and body:

$$
\mathbf{F}(s, t)=\rho S \mathbf{g}-v \dot{\mathbf{r}}^{\mathrm{SH}}(s, \mathbf{q})+\mathbf{F}^{\mathrm{i}}(s, t),
$$

where $\mathbf{F}(s, t)$ is the total external force applied to the rod per unit length, $\rho S$ is the mass of the rod per unit length, and $\mathbf{g}$ is the acceleration of gravity. The interaction forces $\mathbf{F}^{\mathrm{i}}$ are computed using the model presented shortly in Section 4.

The three energies in the equations of motion (4) that are relevant for an elastic rod are:

$$
\begin{gathered}
T(\mathbf{q}, \dot{\mathbf{q}}, t)=\frac{1}{2} \int_{0}^{L} \rho S\left(\dot{\mathbf{r}}^{\mathrm{SH}}(s, \mathbf{q})\right)^{2} \mathrm{~d} s \\
U(\mathbf{q}, t)=\frac{1}{2} \int_{0}^{L} \sum_{i=0}^{2}(E I)_{i}\left(\kappa_{i}^{\mathrm{SH}}(s, \mathbf{q})-\kappa_{i}^{\mathrm{n}}(s)\right)^{2} \mathrm{~d} s \\
D(\mathbf{q}, \dot{\mathbf{q}}, t)=\frac{1}{2} \int_{0}^{L} \gamma \sum_{i=0}^{2}\left(\dot{\kappa}_{i}^{\mathrm{SH}}(s, \mathbf{q})\right)^{2} \mathrm{~d} s .
\end{gathered}
$$

The kinetic energy $T$ is defined in terms of the rod velocity, $\dot{\mathbf{r}}=$ $\mathrm{d} \mathbf{r} / \mathrm{d} t$ in the classical way. The internal energy $U$ in equation $(5 \mathrm{c})$ is the elastic energy of a rod, as derived, for instance, in [Audoly and Pomeau 2006] and used in [Bertails et al. 2005b]. The coefficients $(E I)_{i}$ are the principal bending stiffness of the rod in the directions $\mathbf{n}_{i}$ (for $\left.i=1,2\right)$ while $(E I)_{0}$ is the torsional stiffness, classically written $\mu J$ (for $i=0$ ). These parameters are given by textbook formulas in terms of the material properties (Young's modulus and Poisson's ratio) and of the geometry of the cross-section. The quantities $\kappa_{i}^{\mathrm{n}}(s)$ are called the natural curvatures $(i=1,2)$ and twist $(i=0)$ of the rod. They characterize the shape of the rod in the absence of external force: for $\kappa_{i}(s)=\kappa_{i}^{\mathrm{n}}(s)$ the elastic energy is vanishing and therefore minimum. Vanishing natural curvatures ( $\kappa_{\alpha}^{\mathrm{n}}=0$ for $\left.\alpha=1,2\right)$ model straight hair. Nonzero values will result in wavy, curly or fuzzy hair. In practice, tuning these parameters allows one to choose for the desired hair style, as explained in Section 2. Overall, the mechanical properties of the rod are captured by only six entities, the stiffnesses $\left(E I_{i}\right)_{i=0,1,2}$ and the natural twist and curvatures $\left(\kappa_{i}^{\mathrm{n}}(s)\right)_{i=0,1,2}$. We neglect the dependence of the stiffnesses on $s$, but not that of the natural twist and curvatures: we found that slight variations of $\left(\kappa_{i}^{\mathrm{n}}(s)\right)_{i}$ with $s$ allow for more realistic hair styles. Finally, we choose for the dissipation energy $D$ in equation (5d) a simple heuristic model for capturing visco-elastic effects in hair strands, the coefficient $\gamma$ being the internal friction coefficient.
All the terms needed in equation (4) have been given in equations (5). By plugging the latter into the former, one arrives at explicit equations of motion for the generalized coordinate $\mathbf{q}(t)$. Although straightforward in principle, this calculation is involved ${ }^{3}$. It can nevertheless be worked out easily using a symbolic calculation language such as Mathematica [Wolfram 1999]: the first step is to implement the reconstruction of Super-Helices as given in Appendix $\mathrm{A}$; the second step is to work out the right-hand sides of equations (5), using symbolic integration whenever necessary; the final step is to plug everything back into equation (4). This leads to the equation of motion of a Super-Helix:

$$
\mathbb{M}[s, \mathbf{q}] \cdot \ddot{\mathbf{q}}+\mathbb{K} \cdot\left(\mathbf{q}-\mathbf{q}^{\mathrm{n}}\right)=\mathbf{A}[t, \mathbf{q}, \dot{\mathbf{q}}]+\int_{0}^{L} \mathbf{J}_{i Q}[s, \mathbf{q}, t] \cdot \mathbf{F}^{\mathrm{i}}(s, t) \mathrm{d} s .
$$

In this equation, the bracket notation is used to emphasize that all functions are given by explicit formula in terms of their arguments.

In equation (6), the inertia matrix $\mathbb{M}$ is a dense square matrix of size $3 N$, which depends nonlinearly on $\mathbf{q}$. The stiffness matrix $\mathbb{K}$ has the same size, is diagonal, and is filled with the bending and torsional stiffnesses of the rod. The vector $\mathbf{q}^{\mathrm{n}}$ defines the rest position in generalized coordinates, and is filled with the natural twist or curvature $\kappa_{i}^{\mathrm{n}}$ of the rod over element labelled $Q$. Finally, the vector A collects all remaining terms, including air drag and visco-elastic dissipation, which are independent of $\ddot{\mathbf{q}}$ and may depend nonlinearly on $\mathbf{q}$ and $\dot{\mathbf{q}}$.

\subsection{Time discretization}

The equation of motion (6) is discrete in space but continuous in time. For its time integration, we used a classical Newton semiimplicit scheme with fixed time step. Both the terms $\ddot{\mathbf{q}}$ and $\mathbf{q}$ in the left-hand side are implicited. Every time step involves the solution of a linear system of size $3 N$. The matrix of this linear system is square and dense, like $\mathbb{M}$, and is different at every time step: a conjugate-gradient algorithm is used. The density of $\mathbb{M}$ is the price to be paid for incorporating the inextensibility constraint into the parameterization. It results in degrees of freedom that are non local in physical space.

\subsection{Super-Helices for solving the Kirchhoff equa- tions}

The equations of motion for dynamic elastic rods were derived by Kirchhoff in 1859. A modern derivation of these equations can be found, for instance, in [Audoly and Pomeau 2006]: it follows the same principles as the one for a Super-Helix. The main difference is that we have constrained the material curvatures and twists to be piecewise constant functions of $s$ in equation (3); these functions may depend arbitrarily on $s$ for regular Kirchhoff rods. Apart from this difference, the Super-Helix model is based on the same physical assumptions as the Kirchhoff equations. Therefore, the SuperHelix method provides a discrete model for solving the Kirchhoff equations.

We derived the Super-Helix model after we extensively tested existing integration schemes for the Kirchhoff equations, and eventually realized that they were not well suited for computer graphics applications. We implemented an elegant algorithm, due to [Hou et al. 1998], based on a discretization of these equations by finite differences (this model is referred to as the 'nodal approach' in the video). In this paper, Hou et al. discuss very clearly the difficulties

\footnotetext{
${ }^{3}$ The elements of $\mathbb{M}$, for instance, $\operatorname{read} M_{i Q, i^{\prime} Q^{\prime}}=\frac{1}{2} \iint \mathbf{J}_{i Q}(s, \mathbf{q})$ $\mathbf{J}_{i^{\prime} Q^{\prime}}\left(s^{\prime}, \mathbf{q}\right) \mathrm{d} s \mathrm{~d} s^{\prime}$ where $\mathbf{J}$ is the gradient of $\mathbf{r}^{\mathrm{SH}}(s, \mathbf{q})$ with respect to $\mathbf{q}$.
} 
associated with the numerical integration of the Kirchhoff equations, which are numerically very stiff. They propose an attempt for removing this stiffness. It brings a very significant improvement over previous methods but we found that it was still insufficient for hair animation purposes: there remain quite strong constraints on the time steps compatible with numerical stability of the algorithm. For instance, simulation of a $10 \mathrm{~cm}$ long naturally straight hair strand using the algorithm given in [Hou et al. 1998] remained unstable even with 200 nodes and a time step as low as $10^{-5} \mathrm{~s}$. The stiffness problems in nodal methods have been analyzed in depth by [Baraff and Witkin 1992] who promoted the use of Lagrangian deformable models (sometimes called 'global models' as opposed to nodal ones). This is indeed the approach we used above to derive the Super-Helix model, in the same spirit as [Witkin and Welch 1990; Baraff and Witkin 1992; Qin and Terzopoulos 1996].

We list a few key features of the Super-Helix model which contribute to realistic, stable and efficient hair simulations. All space integrations in the equations of motion are performed symbolically off-line, leading to a quick and accurate evaluation of the coefficients in the equation of motion at every time step. The inextensibility constraint, enforced by equations $(1 \mathrm{a}-1 \mathrm{~b})$, is incorporated into the reconstruction process. As a result, the generalized coordinates are free of any constraint and the stiff constraint of inextensibility has been effectively removed from the equations. Moreover, the method offers a well-controlled space discretization based on Lagrangian mechanics, leading to stable simulations even for small $N$. For $N \rightarrow \infty$, the Kirchhoff equations are recovered, making the simulations very accurate. By tuning the parameter $N$, one can freely choose the best compromise between accuracy and efficiency, depending on the complexity of hair motion and on the allowed computational time. We are aware of another Lagrangian model ${ }^{4}$ used in computer graphics that provides an adjustable number of degrees of freedom, namely the Dynamic NURBS model [Qin and Terzopoulos 1996], studied in the 1D case by [Nocent and Remion 2001]. Finally, external forces can have an arbitrary spatial dependence and do not have to be applied at specific points such as nodes, thereby facilitating the combination with the interaction model.

\section{Application and validation}

In this section, our Super-Helix model is used to animate sparse guide strands that define global hair motion, in a similar way to [Daldegan et al. 1993; Chang et al. 2002]. A new scheme is first proposed for convincingly modelling a hair assembly from this sparse set of guide strands. Then, we provide a validation of our physical model against a series of experiments on real hair, and demonstrate that the Super-Helix model accurately simulates the motion of hair. Images and videos showing our set of results are available at http://wwwevasion.imag.fr/Publications/2006/BACQLL06/.

\subsection{Modelling a hair assembly}

Sparse set of guide hair strands: Realistically animating hair from only a few hundreds of simulated strands is made possible by the local coherence of hair motion. As in previous approaches, the present model aims at mimicking the collective behavior of hair by setting up adequate interaction forces between the simulated strands (Super-Helices) and by adding extra strands at the rendering stage.

\footnotetext{
${ }^{4}$ In this model, geometric parameters, defined by the NURBS control points and the associated weights, are used as generalized coordinates in the Lagrangian formalism. In contrast, we opt here for mechanically-based generalized coordinates: they are the values of the material curvatures and twist, which are the canonical unknowns of the Kirchhoff equations.
}

In the following, we briefly explain how hair interactions are handled, and propose a unified scheme for generating the hair geometry from the set of sparse guide strands.

Hair interactions: Simulating a full head of hair requires an efficient and accurate scheme for handling hair-hair and hair-body collisions. Detection is efficiently processed by exploiting temporal coherence, as in [Raghupathi et al. 2003]: we avoid the quadratic cost of computing proximity of guide strands by keeping track of pairs of closest points over time. As in [Choe et al. 2005], contacts between hair volumes are handled by dissipative penalty forces.

Generating the rendered hair geometry: To be able to handle both smooth and clumpy hairstyles, we avoid choosing between continuum and wisp-based representations for hair. Many real hairstyles display an intermediate behavior with hair strands being more evenly spaced near the scalp than near the tips. Our solution is based on a semi-interpolating scheme to generate non-simulated hair strands from the guide strands (see Figure 4, and the video): we range from full interpolation to generate the extra hair strands near the scalp to no interpolation within a hair wisp near the tips. The separation strongly depends on the level of curliness: straight hair requires more interpolation than curly and clumpy hair. Note that for smooth, interpolated hair, we avoid interpolation between two guide strands having close roots but distant tips by adding a criterion on the distance between tips, see Figure 4, (d).
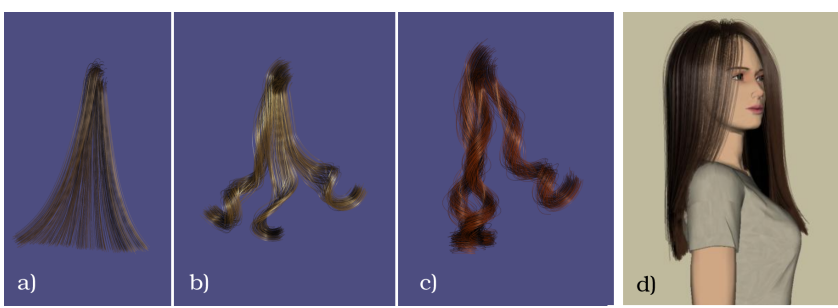

Figure 4: Semi-interpolating scheme for generating the final hair geometry: hair a) is smoothly interpolated, b) is interpolated near the roots but clumpy near the tips, c) forms disjoint locks (no interpolation); d) interpolation across the right shoulder is prevented by the criterion on the maximal distance between the tips.

In our animations, the final hair geometry was rendered using the model of Marschner et al. [2003] for accurately shading a hair strand, together with the algorithm of Bertails et al. [2005a] for casting self-shadows inside hair.

\subsection{Choosing the parameters of the model}

In our model, each Super-Helix stands for an individual hair strand placed into a set of neighboring hair strands, called hair clump, which is assumed to deform continuously. To simulate the motion of a given sample of hair, which can either be a hair wisp or a full head of hair, we first deduce the physical and geometric parameters of each Super-Helix from the structural and physical properties of the hair strands composing the clump. Then, we adjust friction parameters of the model according to the damping observed in real motion of the clump. Finally, interactions are set up between the Super-Helices to account for contacts occurring between the different animated hair groups. In this section, we explain how we set all the parameters of the Super-Helix model using simple experiments performed on real hair. 
Hair mass and stiffness: We set the density $\rho$ to be equal to a typical value for hair, $1.3 \mathrm{~g} \cdot \mathrm{cm}^{-3}$. The mean radius $r$ and the ellipticity $e=\frac{r_{\max }}{r_{\min }}$ of the Super-Helix cross-section are deduced by direct microscopic observation of real hair fibers (see Figure 5, left) whereas Young's modulus and Poisson's ratio are taken from existing tables, which report values for various ethnic origins [Robbins 2002]. These parameters are then used to compute the bending and torsional stiffnesses $(E I)_{i=0,1,2}$ of the Super-Helix, as given by textbook formulas.

Natural curliness: The natural curvatures and twist parameters of the Super-Helix model are set by:

$$
\kappa_{1}^{\mathrm{n}}=1 / r_{\mathrm{h}} \quad \kappa_{2}^{\mathrm{n}}=0 \quad \tau^{\mathrm{n}}=\frac{\Delta_{\mathrm{h}}}{2 \pi r_{\mathrm{h}}^{2}},
$$

where $r_{\mathrm{h}}$ is the radius and $\Delta_{\mathrm{h}}$ the step of the approximate helical shape of the real hair clump, measured near the tips (see Figure 5, right). Indeed, the actual curvatures and twist should be equal to their natural value at the free end of the rod, where the role of gravity becomes negligible. In practice, we add small random variations to these values along each Super-Helix to get more natural results. We have noted that in reality, most hair types have an almost zero natural twist $\tau^{\mathrm{n}}$, except African hair (see Appendix B).
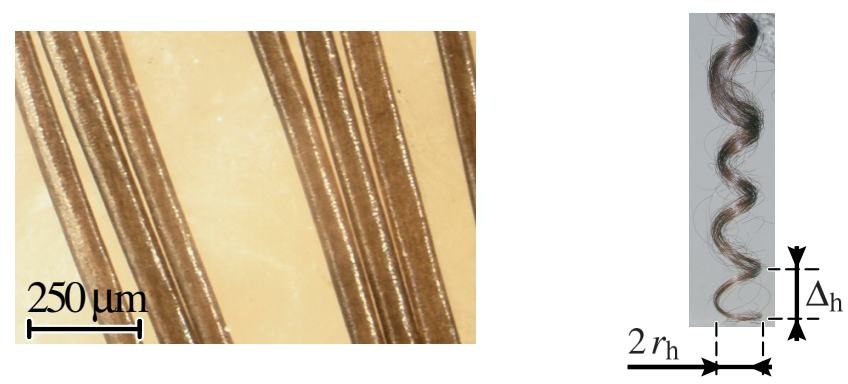

Figure 5: Left, measuring the mean radius $r$ and the ellipticity $e$ of the model by observation of real hair fibers with a videomicroscope. Right, measuring the radius $r_{\mathrm{h}}$ and the step $\Delta_{\mathrm{h}}$ of the natural helical shape at the tip of a real hair clump.

Internal friction $\gamma$ : This parameter measures the amount of internal dissipation within a Super-Helix during motion. It especially accounts for the hair-hair dissipative interactions occurring inside the hair clump whose motion is guided by the Super-Helix. We found that, in practice, the internal friction can be easily adjusted by comparing the amplitude of deformation between the real and the simulated hair clump when a vertical oscillatory motion is imposed, see Figure 6. Typically, we obtained best results with $\gamma \in\left[5.10^{-10}, 5.10^{-11}\right] \mathrm{kg} \cdot \mathrm{m}^{3} \cdot \mathrm{s}^{-1}$.

Air-hair friction coefficient: Once parameter $\gamma$ is chosen, the air-hair friction parameter can be fitted by comparing the damping duration between the real and the simulated hair clump, for example when imposing a pendulum motion. We noted the air-hair friction parameter is strongly related to the local alignment of neighboring hair strands, called the hair discipline in the field of cosmetics. As shown in the video, fuzzy hair is more subject to air damping than regular, disciplined hair. In practice, we chose the air-hair friction coefficient $v$ between $5.10^{-6} \mathrm{~kg} \cdot(\mathrm{m} \cdot \mathrm{s})^{-1}$ (disciplined hair) and $5.10^{-5} \mathrm{~kg} \cdot(\mathrm{m} \cdot \mathrm{s})^{-1}$ (fuzzy hair).

Friction with another object: Contacts between hairs, and between our hair model and external objects (such as the body) are
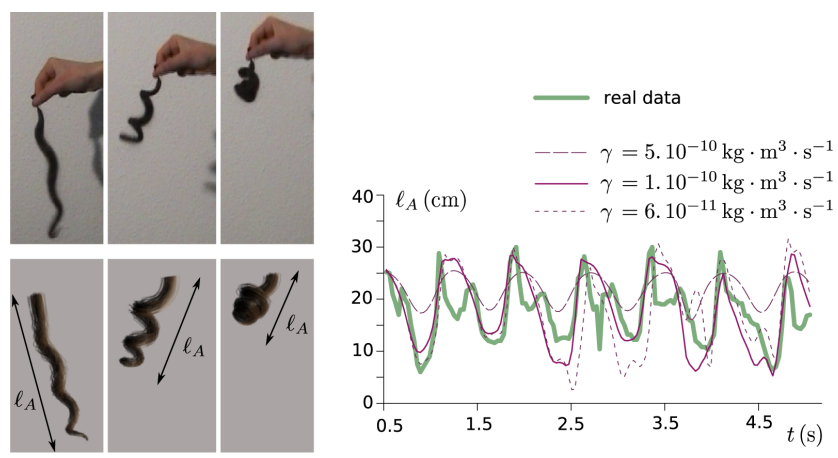

Figure 6: Fitting $\gamma$ for a vertical oscillatory motion of a disciplined, curly hair clump. Left, comparison between the real (top) and virtual (bottom) experiments. Right, the span $\ell_{A}$ of the hair clump for real data is compared to the simulations for different values of $\gamma$. In this case, $\gamma=1.10^{-10} \mathrm{~kg} \cdot \mathrm{m}^{3} \cdot \mathrm{s}^{-1}$ gives qualitatively similar results.

performed through penalty forces which include a normal elastic response together with a tangential viscous friction force. For simulating realistic contacts between hair and external objects, we use an anisotropic friction force, which accounts for the oriented scales covering individual hair fibers. The friction parameter is directly adjusted from real observations of sliding contacts between the hair clump and a given material, and then multiplied by a cosine function to account for the orientation of hair fibers with respect to their sliding motion over the external object. Our video shows some examples of simulated contacts between a hair clump and some fabrics of various roughnesses.

\subsection{Visual comparisons}

Hair clumps: With simulation we have reproduced a series of real experiments on smooth and wavy hair clumps to show that our model captures the main dynamic features of natural hair. We used the technique presented previously to fit the parameters of the Super Helix from the real manipulated hair clump. As shown in Figure 7,
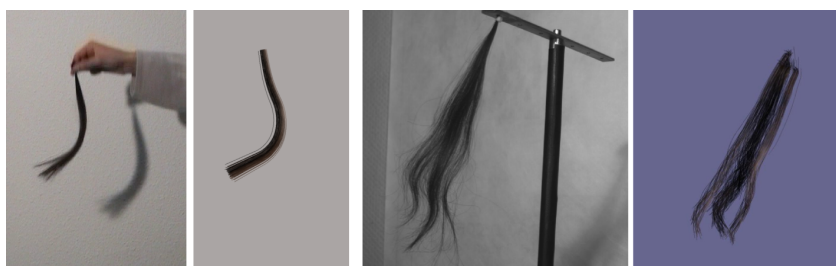

Figure 7: Left, buckling effect caused by vertical oscillations of a hair clump. Right, hair-hair interactions during fast motion.

left, and in the video, our Super-Helix model adequately captures the typical nonlinear behavior of hair (buckling, bending-twisting instabilities), as well as the nervousness of curly hair when submitted to high speed motion (see Figure 6, left). These experiments also allowed us to check the stability of the simulation, even for high speed motion.

Full head of hair: We validated our collective hair behavior model by comparing the details of the motion of a large real wisp with that of a simulated hair volume controlled by three guide strands. Our simulation was able to reproduce closely the motion and the cohesion between neighboring strands (see Figure 7, right, 
and our video). Finally, Figure 8 demonstrates that our model convincingly captures the complex effects occurring in a full head of hair submitted to a high speed shaking motion.
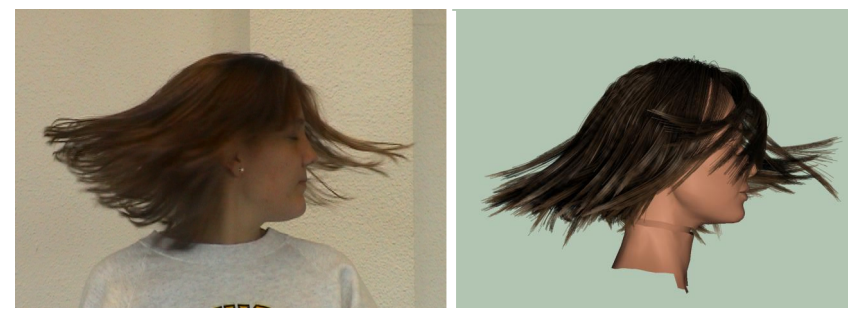

Figure 8: Comparison between a real full head of hair and our model, on a head shaking motion (straight and clumpy hair type).

\subsection{Results and simulation performance}

Figure 1 and the last part of our video show three examples of motion for a full head of hair. Different hair types were simulated, from long to short and curly to straight. To set up our simulations, we used typical parameter values for real hair of different ethnic origins. These parameters are given in Appendix B. We used one hundred guide strands for the wavy and curly hairstyles, and two hundred for the smooth Asian hairstyle.

For all hair types, even long or curly ones, we found it to be unnecessary to use more than 5 to 10 helical elements per guide hair strand. For higher values of $N$, the increase in accuracy becomes imperceptible.

Our model was tested on a $3 \mathrm{GHz}$ Pentium 4 processor. Up to 10 strands can be simulated in real-time. When simulating a full head of hair, we obtained a very reasonable mean computational time of $0.3 \mathrm{~s}$ to $3 \mathrm{~s}$ per frame. The performance of our implementation is thus as good as other recent approaches, such as [Choe et al. 2005]. This is due to the stability of the Super-Helix model, which allows time steps of $\approx 1 / 30 \mathrm{~s}$, even during high speed motion, and to the high order of interpolation provided by the helices, which helps to keep $N$ small while offering a good accuracy.

\subsection{Limitations and future work}

The Super-Helix model remains stable for any number $N$ of helical elements in guide strands. However, the matrix $\mathbb{M}$ used in the dynamic computation is dense, and as a result, the computation time increases quickly with $N$, as $\mathscr{O}\left(N^{2}\right)$. This quadratic time complexity prevents the use of Super-Helices for a very fine simulation. However, this proves to be a minor concern for hair animation purposes, as we find $N$ does not have to be very large for generating pleasant visual results. Moreover, once the number of helical parts is chosen, the complexity of the whole simulation remains linear with respect to the number of guide strands.

Besides this, constraints are currently treated using penalty methods. Analytical methods would be very useful, as they would allow solid friction to be handled. This is one of the planned future extensions of the model.

Although we could advance in the understanding on collective hair behavior, not enough data were available for us to set up the really strong model we would have dreamed of. Indeed, processing nonsimulated hair strands by a simple interpolating scheme between a fixed set of sparse guide hair strands may lose fine-scale details; moreover, when thin objects interact with such sparse hair strands, the coarse granularity of hair may become obvious and distracting.
Quantifying the tendency of hair to cluster and separate according to the hair type as well as to the collisions occurring between hair and external objects would be a very interesting avenue for future work. The relationship between this and the intuitive notions of curliness and discipline could be investigated.

\section{Conclusion}

This paper introduced a deformable model able to simulate hair dynamics for a wide range of hair types, capturing the complex motions observed in real hair motions. In particular, the simulation of curly hair, a notoriously difficult problem, has been demonstrated. Super-Helices are based on Kirchhoff equations for elastic, inextensible rods and on Lagrangian dynamics, and provide a freely adjustable number of degrees of freedom. They take into account important hair features such as the natural curvature and twist of hair strands, as well as the oval shape of their cross section. To stress on the powerful representation of moving hair by Super-Helices, we have presented a rigorous validation of this model, supported by a series of comparative experiments on real hair. We also noted that Super-Helices are able to achieve realistic motions at a very reasonable computational cost: this is permitted by the stability of the method, which enables large time steps, and by the high order of interpolation provided by the helices.

An interesting direction for future research would be to adapt our hair model to a real-time framework, in order to perform interactive hair-styling operations or to use it for character animation in video-games. We could think of setting up an adaptive version of the Super-Helices model, where the number of helical parts would automatically vary over time according to the current deformation and to the available computational power, following work in articulated body dynamics [Redon et al. 2005].

The Super-Helix model was invented and implemented by Basile Audoly. Collective effects were modelled and implemented by Florence Bertails who also undertook the validation of the model through experiments and produced the hair animation results. Prior to this, nodal models were implemented and assessed jointly by Basile Audoly and Florence Bertails.

Acknowledgments This work was supported by L'Oréal. We thank Bertrand Nedellec from L'Oréal Labs for providing us with microscopic measures on real hair samples; Steve Marschner for letting us use his rendering code; Alba Ferrer Biosca and Antoine Bouthors for their time during video acquisition; Paul Kry and Jamie Wither for their useful suggestions for improving the paper. Finally, we would like to thank the anonymous reviewers for their helpful comments.

\section{References}

Anjyo, K., Usami, Y., And Kurihara, T. 1992. A simple method for extracting the natural beauty of hair. 111-120.

Audoly, B., And Pomeau, Y. 2006. Elasticity and Geometry: from hair curls to the nonlinear response of shells. Oxford University Press, Oxford, UK. To appear.

Bando, Y., Chen, B.-Y., And Nishita, T. 2003. Animating hair with loosely connected particles. Computer Graphics Forum (Eurographics'03) 22, 3, 411-418.

BARAFF, D., AND WitKIn, A. 1992. Dynamic simulation of non-penetrating flexible bodies. ACM Computer Graphics (SIGGRAPH'92) 26, 2, 303-308. 
Bertails, F., Kim, T.-Y., Cani, M.-P., and Neumann, U. 2003. Adaptive wisp tree - a multiresolution control structure for simulating dynamic clustering in hair motion. In $A C M S I G$ GRAPH Symposium on Computer Animation, 207-213.

Bertails, F., Ménier, C., And CAni, M.-P. 2005. A practical self-shadowing algorithm for interactive hair animation. In Proc. Graphics Interface, 71-78.

Bertails, F., Audoly, B., Querleux, B., Leroy, F., LÉvÊQUe, J.-L., AND CANI, M.-P. 2005. Predicting natural hair shapes by solving the statics of flexible rods. In Eurographics '05 (short papers).

Chang, J., Jin, J., AND Yu, Y. 2002. A practical model for hair mutual interactions. In ACM SIGGRAPH Symposium on Computer Animation, 73-80.

Choe, B., Choi, M., And Ko, H.-S. 2005. Simulating complex hair with robust collision handling. In ACM SIGGRAPH Symposium on Computer Animation, 153-160.

Daldegan, A., Thalmann, N. M., Kurihara, T., And THALMANN, D. 1993. An integrated system for modeling, animating and rendering hair. Computer Graphics Forum 12, 3, 211-221.

Hadap, S., And Magnenat-Thalmann, N. 2001. Modeling dynamic hair as a continuum. Computer Graphics Forum (Eurographics'01) 20, 3, 329-338.

HoU, T., KlapPer, I., AND SI, H. 1998. Removing the stiffness of curvature in computing 3-d filaments. J. Comput. Phys. 143, $628-664$.

KoH, C., And Huang, Z. 2001. A simple physics model to animate human hair modeled in $2 \mathrm{D}$ strips in real time. In $E G$ CAS '01, 127-138.

LEE, D.-W., AND Ko, H.-S. 2001. Natural hairstyle modeling and animation. Graphical Models 63, 2 (March), 67-85.

Lindelof, B., Forslind, B., Hedblad, M., And Kaveus, U. 1988. Human hair form. morphology revealed by light and scanning electron microscopy and computer aided three-dimensional reconstruction. Arch. Dermatol. 124, 9, 1359-1363.

Marschner, S., Jensen, H., Cammarano, M., Worley, S., AND HANRAHAN, P. 2003. Light scattering from human hair fibers. ACM Transactions on Graphics (SIGGRAPH'03) 22, 3 (July), 281-290.

Nocent, O., AND Remion, Y. 2001. Continuous deformation energy for dynamic material splines subject to finite displacements. In EG CAS '01, 87-97.

PAI, D. 2002. Strands: Interactive simulation of thin solids using cosserat models. Computer Graphics Forum (Eurographics'02) $21,3,347-352$.

Plante, E., Cani, M.-P., And Poulin, P. 2001. A layered wisp model for simulating interactions inside long hair. In $E G$ CAS '01, Springer, Computer Science, 139-148.

Qin, H., AND Terzopoulos, D. 1996. D-nurbs: A physicsbased framework for geometric design. IEEE Trans. on Visualization and Computer Graphics 2, 1, 85-96.

Raghupathi, L., Cantin, V., Faure, F., and Cani, M.-P. 2003. Real-time simulation of self-collisions for virtual intestinal surgery. In International Symposium on Surgery Simulation and Soft Tissue Modeling, Springer-Verlag, no. 2673 in Lecture Notes in Computer Science, 15-26.
Redon, S., Galoppo, N., And Lin, M. 2005. Adaptive dynamics of articulated bodies. ACM Transactions on Graphics (SIGGRAPH'05) 24, 3, 936-945.

Robiins, C. 2002. Chemical and Physical Behavior of Human Hair. 4th ed. Springer.

Rosenblum, R., Carlson, W., And Tripp, E. 1991. Simulating the structure and dynamics of human hair: Modeling, rendering, and animation. The Journal of Visualization and Computer Animation 2, 4, 141-148.

WARD, K., AND LIN, M. C. 2003. Adaptive grouping and subdivision for simulating hair dynamics. In Proceedings of Pacific Graphics'03, 234-243.

WitKin, A., AND WelCh, W. 1990. Fast animation and control of non-rigid structures. ACM Computer Graphics (SIGGRAPH'90) 24, 4, 243-252.

Wolfram, S. 1999. The Mathematica book (4th edition). Cambridge University Press, New York, NY, USA.

\section{A Helical solution}

We show here that the reconstruction of the rod can be carried out over any particular element $S_{Q}=\left[s_{Q}^{\mathrm{L}}, s_{Q}^{\mathrm{R}}\right]$ of the Super-Helix, over which the functions $\left(\kappa_{i}(s)\right)_{i}$ are constant by construction. By equations (1), $\boldsymbol{\Omega}^{\prime}=\sum_{i} \kappa_{i}^{\prime} \mathbf{n}_{i}+\boldsymbol{\Omega} \times \mathbf{\Omega}=\mathbf{0}$, which means that the Darboux vector is constant along each element. For a given element $Q$, let us therefore introduce $\Omega$ the norm of the vector $\boldsymbol{\Omega}$ and $\boldsymbol{\omega}=\boldsymbol{\Omega} / \Omega$ the unit vector aligned with $\boldsymbol{\Omega}$ (the case $\boldsymbol{\Omega}=\mathbf{0}$ is considered separately, see below). Finally, we write $\mathbf{a}^{\|}=(\mathbf{a} \cdot \boldsymbol{\omega}) \boldsymbol{\omega}$ and $\mathbf{a}^{\perp}=\mathbf{a}-\mathbf{a}^{\|}$as the projection of an arbitrary vector $\mathbf{a}$ parallel to and perpendicular to the axis spanned by $\omega$, respectively.

Since $\boldsymbol{\Omega}$ is constant, integration of equation (1b) over an element is straightforward. The material frame 'rotates' around $\boldsymbol{\omega}$ with a constant rate of rotation $\Omega$ per unit of curvilinear length. Therefore, the material frame at coordinate $s \in S_{Q}$ is obtained from the material frame $\mathbf{n}_{i, \mathrm{~L}}^{Q}=\mathbf{n}_{i}\left(s_{Q}^{\mathrm{L}}\right)$ given on the left-hand side of the interval $S_{Q}$, by a rotation with angle $\Omega\left(s-s_{Q}^{\mathrm{L}}\right)$ and axis parallel to $\boldsymbol{\omega}$ :

$$
\mathbf{n}_{i}(s)=\mathbf{n}_{i, \mathrm{~L}}^{Q \|}+\mathbf{n}_{i, \mathrm{~L}}^{Q \perp} \cos \left(\Omega\left(s-s_{\mathrm{L}}^{Q}\right)\right)+\boldsymbol{\omega} \times \mathbf{n}_{i, \mathrm{~L}}^{Q \perp} \sin \left(\Omega\left(s-s_{\mathrm{L}}^{Q}\right)\right) .
$$

By equation (1a), the centerline $\mathbf{r}(s)$ is then found by spatial integration of $\mathbf{n}_{0}(s)$ :

$\mathbf{r}(s)=\mathbf{r}_{\mathrm{L}}^{Q}+\mathbf{n}_{0, \mathrm{~L}}^{Q \|}\left(s-s_{\mathrm{L}}^{Q}\right)+\mathbf{n}_{0, \mathrm{~L}}^{Q \perp} \frac{\sin \left(\Omega\left(s-s_{\mathrm{L}}^{Q}\right)\right)}{\Omega}+\boldsymbol{\omega} \times \mathbf{n}_{0, \mathrm{~L}}^{Q \perp} \frac{1-\cos \left(\Omega\left(s-s_{\mathrm{L}}^{Q}\right)\right)}{\Omega},(7 \mathrm{~b})$

where $\mathbf{r}_{\mathrm{L}}^{Q}=\mathbf{r}\left(s_{Q}^{\mathrm{L}}\right)$ is the prescribed position of the centerline on the left-hand side of the interval. Equations (7) provide the explicit reconstruction of an element. Its centerline is a helix with axis parallel to $\boldsymbol{\omega}$. An equivalent derivation based on Rodrigues' formula is given in [Pai 2002]. Two degenerate cases are possible and must be considered separately: the curve is an arc of circle when $\tau=0$ and $\kappa_{1} \neq 0$ or $\kappa_{2} \neq 0$; it is a straight line when $\kappa_{1}=\kappa_{2}=0$, which can be twisted $(\tau \neq 0)$ or untwisted $(\tau=0$, implying $\boldsymbol{\Omega}=\mathbf{0})$.

Equations (7) can be used to propagate the centerline and the material frame from the left-hand side $s_{Q}^{\mathrm{L}}$ of the element to its right-hand side $s_{Q}^{\mathrm{R}}$. The whole rod can then be reconstructed by applying this procedure over every element successively, starting from the scalp where $\mathbf{r}$ and $\mathbf{n}_{i}$ are prescribed by equation (1c). This yields explicit formulae for the functions $\mathbf{r}^{\mathrm{SH}}(s, \mathbf{q})$ and $\mathbf{n}_{i}^{\mathrm{SH}}(s, \mathbf{q})$, which have the form of equation (7) over each element. The integration constants are determined by continuity at the element boundaries.

\section{B Parameter values for natural hair}

\begin{tabular}{|l|c|c|c|c|}
\hline & $\begin{array}{c}\text { Asian } \\
\text { (smooth) }\end{array}$ & $\begin{array}{c}\text { Caucasian 1 } \\
\text { (wavy) }\end{array}$ & $\begin{array}{c}\text { Caucasian 2 } \\
\text { (curly) }\end{array}$ & $\begin{array}{c}\text { African } \\
\text { (fuzzy) }\end{array}$ \\
\hline \hline Radius $(\mu \mathrm{m})$ & 50 & 35 & 50 & 50 \\
\hline Ellipticity & 1 & 1.1 & 1.1 & 1.2 \\
\hline Helix radius $(\mathrm{cm})$ & 0 & 1 & 0.6 & 0.1 \\
\hline Helix step $(\mathrm{cm})$ & 0 & 0.5 & 0.5 & 1 \\
\hline Young's mod. $(\mathrm{GPa}$ ) & 1 & 2 & 1.5 & 0.5 \\
\hline Poisson's ratio & 0.48 & 0.48 & 0.48 & 0.48 \\
\hline
\end{tabular}

